\title{
Novel Model of Thermal Conductivity for Optical Materials
}

\author{
Yoichi SATO, Hideki ISHIZUKI, and Takunori TAIRA \\ Laser Research Center for Molecular Science, Institute for Molecular Science, \\ 38 Nishigonaka, Myodaiji, Okazaki 444-8585
}

(Received January 31, 2008)

\begin{abstract}
We have proposed a novel model of thermal conductivity in various optical materials. This numerical model for thermal conductivity requires one material parameter for isovolumic specific heat and two parameters for thermal diffusivity in the calculation of each optical material. The validity of our model was demonstrated and it was adequately able to present thermal conductivities between $25^{\circ} \mathrm{C}$ and $200^{\circ} \mathrm{C}$ in $\mathrm{Y}_{3} \mathrm{Al}_{5} \mathrm{O}_{12}, \mathrm{YVO}_{4}, \mathrm{GdVO}_{4}$, stoichiometric and congruent $\mathrm{LiTaO}_{3}$, and synthetic quartz.
\end{abstract}

Key Words: Laser materials, Thermal conductivity, Thermal diffusivity, Specific heat

\section{Introduction}

Recent progress in the "Giant Micro Photonics" enables an extremely high photon-energy emission from the microscopic volume. ${ }^{1)}$ An optical energy density of extracted laser output from a laser material has increased every year, and microchip based active mirror laser allows efficient heat removing for the highly density laser operation up to $200 \mathrm{~kW} / \mathrm{cm}^{3}{ }^{32)}$ Although it would be significant to scale up the brightness of laser output, severe thermal problems prevent further power scaling of laser oscillators. In the case of a high power microchip laser reported in Ref. 2, temperature $(T)$ of laser crystal rose up to $200^{\circ} \mathrm{C}$ due to an excessive heat from optical pumping. Therefore it is necessary for designing of high-power laser cavities to avoid thermal problems by careful heat treatment with precise thermal conductivity $(\kappa)$. Dependence of $\kappa$ on $T$ from room temperature (RT, $=25^{\circ} \mathrm{C}$ ) to $200^{\circ} \mathrm{C}$ should be also included into this thermal management. Although models that can explain temperature dependence already exist, ${ }^{3)}$ a novel model that can give isopiestic specific heat for unit mass $\left(C_{\mathrm{P}}\right)$ and thermal diffusivity $(H)$ separately is necessary for developing laser cavities that have various boundary condition of laser head.

There is no conventional expression of $H$ under temperature range between $\mathrm{RT}$ and $200^{\circ} \mathrm{C}$ besides empirical $1 / T$-law above Debye temperature $\left(\Theta_{\mathrm{D}}\right),{ }^{4}$ ) moreover it is very difficult to measure precise $H$ in heated materials due to the heat losses from the periphery of the sample above RT. ${ }^{5}$ We developed quasi-one-dimensional flash method (Q1DFM) within temperature range from RT to $200^{\circ} \mathrm{C}^{\text {; }}$; besides previous studies on $\kappa$ in laser media have been mainly about low temperature thermal conduction. ${ }^{7,8)}$

In this work we proposed a novel model of $\kappa$ for optical materials, which can describe $\kappa$ by three material parameters. We also showed correspondence between the measured value and modelled value of $\kappa$ in optical materials that have cubic, tetragonal, rhombic, and amorphous structures.

\section{Numerical Model}

The value of $\kappa$ is a product of isovolumic specific heat for unit volume $\left(C_{\mathrm{V}}\right)$, and $H$. While it is difficult to measure $C_{\mathrm{V}}$ directly, the experimental evaluation of $C_{\mathrm{P}}$ is quite easy by differential scanning calorimetry (DSC). $\kappa$ can be expressed by the product of density $(\rho), C_{\mathrm{P}}$, and $H$, because $C_{\mathrm{V}}$ is approximated to be a product of $\rho$ and $C_{\mathrm{P}}$ from Nernst-Lindemann's law expressed by

$$
C_{\mathrm{P}}-\frac{C_{\mathrm{V}}}{\rho}=\frac{T \alpha^{2} E}{3 \rho(1-2 v)}
$$

where $\alpha, E$, and $v$ are thermal expansion coefficient, modulus of elasticity, and Poisson ratio, respectively.

From Debye model of phonon in dielectric materials, $C_{\mathrm{P}}$ can be expressed by molecular mass $(M)$, ion number in a molecule $(m)$, and Debye temperature $\left(\Theta_{\mathrm{D}}\right)$ as

$$
C_{P}=3 N_{\mathrm{A}} k_{\mathrm{B}} \frac{m}{M} f_{D}\left(\frac{T}{\Theta_{\mathrm{D}}}\right)
$$

where $T, N_{\mathrm{A}}$, and $k_{\mathrm{B}}$ are material temperature, Avogadro's constant, and Boltzmann's constant, respectively. Here $f_{\mathrm{D}}(x)$ is Debye function defined by

$$
f_{\mathrm{D}}(x)=3 x^{3} \int_{0}^{\frac{1}{x}} \frac{z^{3} e^{z}}{\left(e^{z}-1\right)^{2}} d z
$$

In the case of $H$ there are many reports on the numerical model, and unfortunately they are useful only in limited conditions. The model where $H$ depends on Plank's distribution $^{8)}$ is applicable only in cryogenic conditions, and the model where $H$ inversely proportional to $T^{4)}$ is useful at higher than $\Theta_{\mathrm{D}}$. In order to explain the geophysical issues, more detailed model containing three thermal parameters was given by ${ }^{9)}$

$$
H(T)=\left[H\left(T_{0}\right)-H_{H}\right]\left(\frac{T_{0}}{T}\right)^{r}+H_{H}
$$

where $T_{0}, H_{\mathrm{H}}$, and $r$ are a representative temperature, a high temperature limit of $H$, and a constant depends on $T_{0}$ and phonon kinetics. However it has been difficult not only to define $r$ and but also to confirm its validity. Because phonon 
Table.1 Crystal parameters of YAG, SLT, CLT, $\mathrm{YVO}_{4}$, $\mathrm{GdVO}_{4}$, and $\mathrm{S}-\mathrm{SiO}_{2}$.

\begin{tabular}{|c|c|c|c|}
\hline \hline & $\rho\left(\mathrm{g} / \mathrm{cm}^{3}\right)$ & $m$ & $M$ \\
\hline $\mathrm{Y}_{3} \mathrm{Al}_{5} \mathrm{O}_{12}$ & 4.55 & 20 & 593.613 \\
\hline $\mathrm{YVO}_{4}$ & 4.22 & 6 & 203.843 \\
\hline $\mathrm{GdVO}_{4}$ & 5.47 & 6 & 272.188 \\
\hline $\mathrm{SLT}$ & 7.44 & 5 & 236.059 \\
\hline $\mathrm{CLT}$ & 7.46 & 5 & 236.059 \\
\hline $\mathrm{S}-\mathrm{SiO}_{2}$ & 2.22 & 3 & 60.083 \\
\hline \hline
\end{tabular}

Table.2 Thermal parameters of YAG, SLT, CLT, $\mathrm{YVO}_{4}$, $\mathrm{GdVO}_{4}$, and $\mathrm{S}-\mathrm{SiO}_{2}$.

\begin{tabular}{|c|c|c|c|}
\hline \hline & $\Theta_{\mathrm{D}}(\mathrm{K})$ & $A\left(\mathrm{~mm}^{2} \mathrm{~K} / \mathrm{s}\right)$ & $B\left(\mathrm{~mm}^{2} / \mathrm{s}\right)$ \\
\hline $\mathrm{Y}_{3} \mathrm{Al}_{5} \mathrm{O}_{12}$ & 795 & 1309.65 & -0.700365 \\
\hline $\mathrm{YVO}_{4}$ a-axis & 718 & 1255.98 & -0.46298 \\
\hline $\mathrm{YVO}_{4}$ c-axis & 718 & 1670.15 & -0.455651 \\
\hline $\mathrm{GdVO}_{4}$ a-axis & 677 & 1261.40 & -0.525256 \\
\hline $\mathrm{GdVO}_{4}$ c-axis & 677 & 1468.06 & -0.466545 \\
\hline $\mathrm{SLT}$ c-axis & 750 & 929.534 & -0.511528 \\
\hline $\mathrm{CLT} \mathrm{c-axis}$ & 750 & 349.311 & 0.225441 \\
\hline $\mathrm{S}-\mathrm{SiO}_{2}$ & 990 & 55.7963 & 0.597186 \\
\hline \hline
\end{tabular}

kinetics are dependent not only on crystal structures but also on crystal qualities, $r$ is a peculiar value in each sample. Moreover an experimental estimation of $r$ requires precise $H$ under wide temperature range from RT to quite high temperature near $H_{\mathrm{H}}$ (generally over $1000^{\circ} \mathrm{C}$ )

In order to obtain a convenient and generalized model for analysis thermal condition of optical materials, we have tried to develop a novel model for $H$ by both fixing $r$ to 1 for all materials and limiting the applicable temperature range. In this assumption $H$ is given by

$$
H(T)=\frac{A}{T}+B
$$

where $A$ and $B$ are fitting parameters. Equation (5) is the same as the first two terms of eq.(4) expanded by Taylor series. This means that assuming $r$ to be 1 is the reason why applicable temperature range in eq.(5) is limited between RT to $200^{\circ} \mathrm{C}$. $B$ is a time-independent component of $H$, and sometimes $B$ become negative as a result of the nonlinear temperature dependence of $H$. Finally, $\kappa$ can be given by

$$
\kappa(T)=3 \rho N_{\mathrm{A}} k_{\mathrm{B}} \frac{m}{M}\left(\frac{A}{T}+B\right) f_{D}\left(\frac{T}{\Theta_{\mathrm{D}}}\right)
$$

There are only three material parameters of $A, B$, and $\Theta_{\mathrm{D}}$ in eq.(6) except what are determined from the crystal structure.

\section{Experimental Setup}

$C_{\mathrm{P}}$ and $H$ was measured with following samples. (i) Cubic material of $\mathrm{Y}_{3} \mathrm{Al}_{5} \mathrm{O}_{12}$ (YAG, synthesized by Scientific Materials). (ii) Tetragonal materials of $\mathrm{YVO}_{4}$ (ITI Electro-Optics) and $\mathrm{GdVO}_{4}$ (Shandong Newphotonics). (iii)

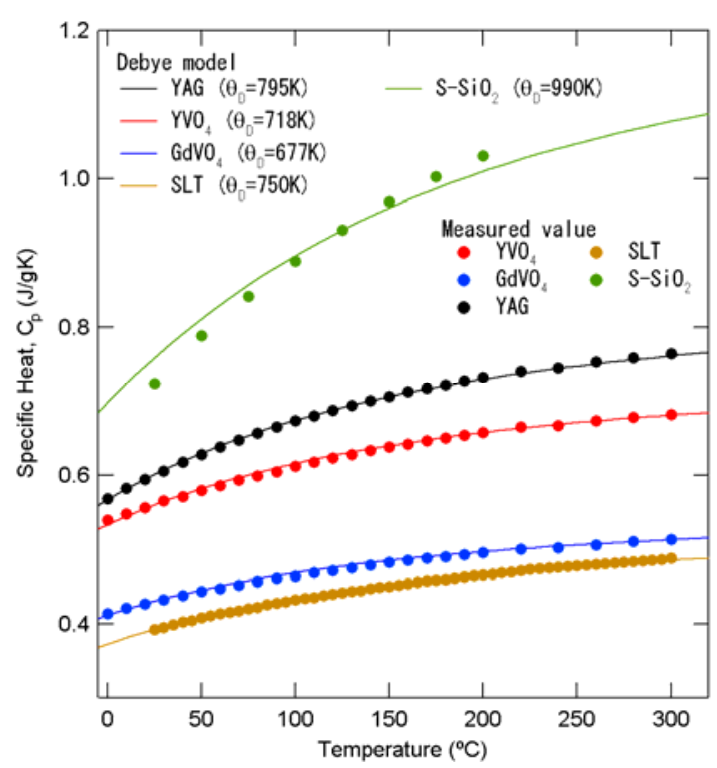

Fig.1 Measured $C_{\mathrm{P}}$ of various optical materials (marker) and simulations by eq.(2) (line). $\Theta_{\mathrm{D}}$ are shown in Table 1, 2.

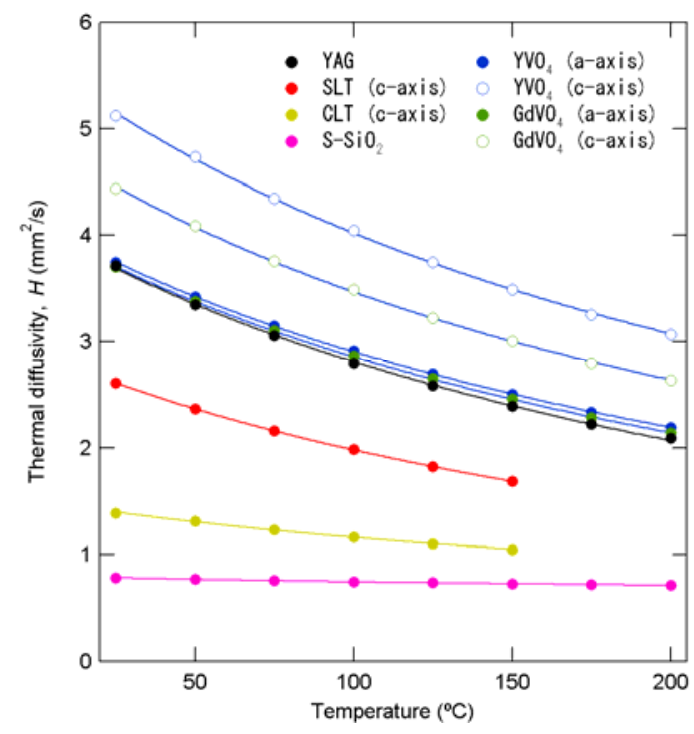

Fig.2 Measured $H$ of various optical materials (marker) and simulations by eq.(5) (line). Fitting parameters are shown in Table 1, 2.

Rhombic materials of stoichiometric $\mathrm{LiTaO}_{3}$ (SLT, OXIDE), and congruent $\mathrm{LiTaO}_{3}$ (CLT, Yamaju ceramics). (iv) Amorphous of synthetic quartz $\left(\mathrm{S}-\mathrm{SiO}_{2}\right)$. All crystal parameters are summarized in Table 1.

We measured $C_{\mathrm{P}}$ of YAG, SLT, CLT, $\mathrm{YVO}_{4}$, and $\mathrm{GdVO}_{4}$ relatively to sapphire standard within the range from 0 to $300^{\circ} \mathrm{C}$ at a heating of $10 \mathrm{~K} / \mathrm{min}$ by DSC (DSC $204 \mathrm{~F} 1$, Netzsch). The sample size is $5.0 \mathrm{~mm}$ in diameter and $1.0 \mathrm{~mm}$ 
in thickness. $C_{\mathrm{P}}$ of $\mathrm{S}-\mathrm{SiO}_{2}$ with the size of $12.7-\mathrm{mm}$ in diameter and 1.0-mm thickness was evaluated from 25 to $200^{\circ} \mathrm{C}$ relatively to YAG by flash method (LFA 447 nanoflash, Netzsch). Error under $C_{\mathrm{P}}$ measurement was below $2 \%$ except by flash method of $7 \%$ from SI-trace.

The measurement of $H$ was carried out by the Q1DFM from 25 to $200^{\circ} \mathrm{C}$ with samples of YAG, SLT, CLT, $\mathrm{YVO}_{4}, \mathrm{GdVO}_{4}$, and $\mathrm{S}_{-} \mathrm{SiO}_{2}$ by flash method (LFA 447 nanoflash, Netzsch). The sample size of cubic, rhombic, tetragonal, and amorphous materials were $\varnothing-12.7 \mathrm{~mm} \times \mathrm{t}-1.0 \mathrm{~mm}, 10 \times 10 \times 1 \mathrm{~mm}^{3}$, $\varnothing-8.0$ $\mathrm{mm} \times \mathrm{t}-1.0 \mathrm{~mm}$, and $\varnothing-12.7 \mathrm{~mm} \times \mathrm{t}-1.0 \mathrm{~mm}$, respectively. Reproducibility of Q1DFM was below 1\%.

\section{Results}

Figures 1 and 2 show the result of the measured $C_{\mathrm{P}}$ and $H$, respectively. $\Theta_{\mathrm{D}}$ of each materials were calculated from $C_{\mathrm{P}}$ at $25^{\circ} \mathrm{C}$ using eq.(2) except $\mathrm{S}-\mathrm{SiO}_{2}$ at $125^{\circ} \mathrm{C}$, and parameters of $A$ and $B$ were determined by minimum square fitting by eq. (5). All fitting parameters shown in Table 2 can reproduce measured $C_{\mathrm{P}}$ and $H$ with the error below $0.5 \%$ except $C_{\mathrm{P}}$ of $\mathrm{S}-\mathrm{SiO}_{2}$. The reason why an error in $C_{\mathrm{P}}$ measurement of $\mathrm{S}-\mathrm{SiO}_{2}$ reached $5 \%$ is due to influences of its inhomogeneous structure.

\section{Discussions}

The model proposed in this work is applicable for only dielectric materials because eq.(2) is based on an assumption where main heat carrier is limited to only lattice phonons. Moreover eq.(2) is not appropriate for opaque materials. Since radiated infra-red photons from a measured sample under high temperature conditions is self-absorbed, photon in opaque samples can be one of heat carriers.

Figure 3 shows the reported value of $H$ in YAG under various temperature range. ${ }^{6-8)}$ Only under cryogenic conditions $H$ was well fitted by the model given by ${ }^{8)}$

$$
H(T)=H\left(\frac{\Theta_{D}}{b}\right) \frac{\exp \left(\frac{\Theta_{D}}{b T}\right)-1}{e-1}
$$

where $b$ is a fitting parameter. High-order phonon-phonon interaction occurs under higher temperature than RT, therefore $b$ become larger than that of under cryogenic condition. It is thought that anincrease of energy dissipation due to high-order phonon-phonon interaction causes temperature dependence of $H$ in optical materials to the empirical $1 / T$-law at quite lower temperature that is far lower than $\Theta_{\mathrm{D}}$.

In order to confirm a validity of eq.(2), it is necessary to evaluate the right side of eq.(1) that is a difference between $C_{\mathrm{V}}$ and $C_{\mathrm{p}}$. For example by using of the most common material $\mathrm{YAG}^{10)}$ this difference was calculated as $1.1 \times 10^{-3} \mathrm{~J} / \mathrm{gK}$. It is less than $0.2 \%$ of $C_{\mathrm{P}}$. This indicates that at least from RT to $200^{\circ} \mathrm{C}$ we can consider $\rho C_{\mathrm{P}}$ to be $C_{\mathrm{V}}$.

Because differences between reported $\Theta_{\mathrm{D}}$ were very large: in the case of YAG from $505 \mathrm{~K}^{11)}$ to $750 \mathrm{~K},{ }^{12)}$ it is important that $\Theta_{\mathrm{D}}$ should be determined from $C_{\mathrm{P}}$ measurement. On the contrary our estimated $\Theta_{\mathrm{D}}$ of YAG was $795 \mathrm{~K}$ that was larger than these reported value. Larger $\Theta_{\mathrm{D}}$ gives smaller $C_{\mathrm{V}}$, which becomes one circumstantial evidence of experiment under adiabatic condition.

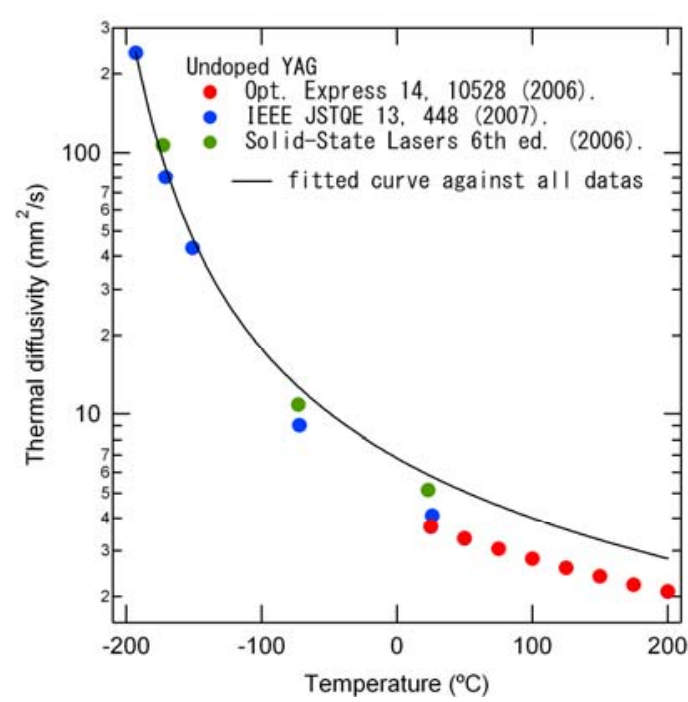

Fig.3 $H$ of YAG under various temperature range ${ }^{6-8)}$ (marker) and simulations by eq.(7) (line). Fitting parameter $b$ was 2.14 .

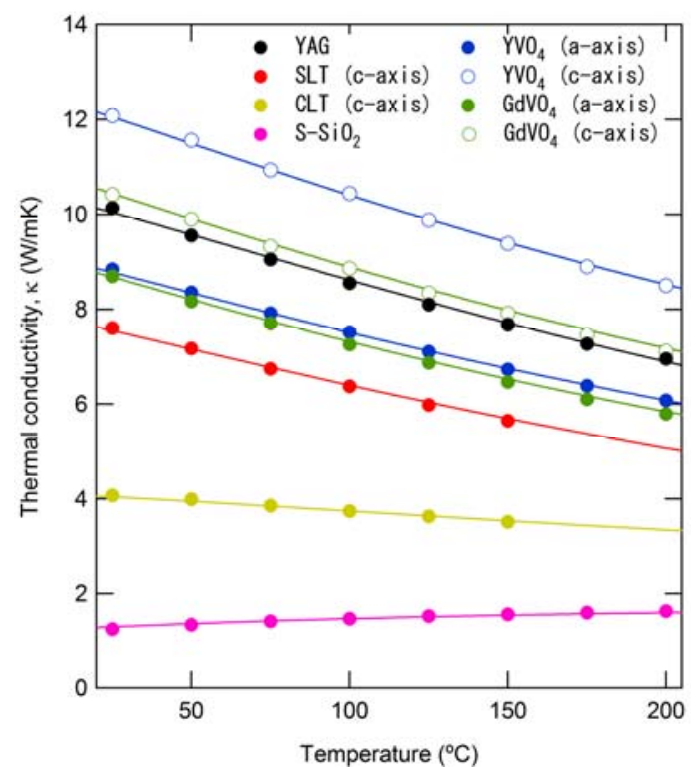

Fig.4 Measured $\kappa$ of various optical materials (marker) and simulations by eq.(6) (line). Fitting parameters are shown in Table $1,2$.

It is enough for optical materials to consider thermal condition from RT to $200^{\circ} \mathrm{C},{ }^{2)}$ and according to our result this temperature relates a quite narrow range within $0.30 \Theta_{\mathrm{D}}$ and $0.70 \Theta_{\mathrm{D}}$, which guarantees the validity of eq.(5).

It was experimentally found that $\Theta_{\mathrm{D}}$ of various materials are constant within the range of 25 to $200^{\circ} \mathrm{C}$, and was also found that even in quite lower temperature than $\Theta_{\mathrm{D}}$ eq.(5) gives adequate temperature dependence. The simulation of $\kappa$ by eq.(6) can reproduce a good agreement with the 
experimentally obtained value as shown in Fig. 4, which indicates that our model proposed in this work is quite useful for the optical design of photonic devices.

\section{Conclusion}

The novel model of a thermal conductivity for optical materials was proposed. We demonstrated that the model containing a few material parameters was adequately able to reproduce $\kappa$ of various optical materials as YAG, SLT, CLT, $\mathrm{YVO}_{4}, \mathrm{GdVO}_{4}$, and $\mathrm{S}-\mathrm{SiO}_{2}$ within a temperature range between $25^{\circ} \mathrm{C}$ and $200^{\circ} \mathrm{C}$. This numerical model is quite useful for not only thermal analysis in laser cavities or optical waveguides but also the evaluation of physical properties in various transparent materials.

\section{Acknowledgements}

This work was partially supported by Genesis Research Institute, Inc., New Energy and Industrial Technology Development Organization (NEDO), and the Special Coordination Funds for Promoting Science and Technology, from the Ministry of Education, Culture, Sports, Science, and
Technology of Japan. Authors would like to thank NETZSCH GmbH for DSC measurement.

\section{References}

1) T. Taira: IEEE J. Sel. Top. Quantum Electron. 13 (2007) 798.

2) M. Tsunekane and T. Taira: Appl. Phys. Lett. 90 (2007) 121101.

3) C. Stewen, K. Contag, M. Larionov, A. Giesen, and H. Hügel: IEEE J. Sel. Top. Quantum Electron. 6 (2000) 650.

4) A. M. Hofmeister: Phys. Chem. Minerals 33 (2006) 45

5) J. E. Parrott and A. D. Stuckes: Thermal conductivity of solids (Pion Limited, London, 1975) P.15

6) Y. Sato and T. Taira: Opt. Express 14 (2006) 10528

7) D. C. Brown: IEEE J. Quantum Electron. 33 (1997) 861.

8) T. Y. Fan, D.J. Ripin, R. L. Aggarwal, J. R. Ochoa, B. Chann, M. Tilleman, and J. Spitzberg: IEEE J. Sel. Top. Quantum Electron. 13 (2007) 448.

9) M. C. Roufosse, and P. G. Klemens: J. Geoph. Res. 79 (1974) 703.

10) W. Koechner: Solid-state laser engineering 6th ed., (Springer Science+Business Media, Inc. Berin, 2006) p.55.

11) M. G. Beghi, C. E. Bottani, and V. Russo: J. Appl. Phys. 87 (2000) 1769.

12) J. M. Pellegrino and W. M. Yen: Phys. Rev. B 24 (1981) 6719. 